\title{
Alzheimer's Disease Results in Salivary Redox Imbalance Towards Oxidation Reactions
}

\author{
Anna Zalewska \\ Medical University of Bialystok \\ Anna Klimiuk \\ Medical University of Bialystok \\ Sara Zięba \\ Medical University of Bialystok \\ Olga Wnorowska \\ Independent Public Mental Health Care Centre in Choroszcz \\ Małgorzata Rusak \\ Medical University of Bialystok \\ Napoleon Waszkiewicz \\ Medical University of Bialystok \\ Izabela Szarmach \\ Medical University of Bialystok \\ Krzysztof Dzierżanowski \\ Independent Public Mental Health Care Centre in Choroszcz \\ Mateusz Maciejczyk ( $\square$ mat.maciejczyk@gmail.com ) \\ Medical University of Bialystok
}

Research Article

Keywords: Alzheimer disease, antioxidants, nitrosative stress, oxidative stress, saliva

Posted Date: September 7th, 2021

DOI: https://doi.org/10.21203/rs.3.rs-871834/v1

License: (a) This work is licensed under a Creative Commons Attribution 4.0 International License. Read Full License 


\section{Abstract}

Alzheimer's disease (AD) is associated with the deposition of $\beta$-amyloid in the brain. $A D$ accounts for over $50 \%$ of cases of dementia which results from disturbances in redox homeostasis. Indeed, increased intensity of protein oxidation and nitration as well as lipid peroxidation is observed in brain areas with considerable amounts of amyloid plaques and neurofibrillary tangles. However, little is known about the oxidoreductive balance of salivary glands in $A D$ patients. Therefore, the aim of this study was to evaluate the antioxidant barrier and oxidative/nitrosative stress biomarkers in stimulated saliva and blood of AD patients. The study was participated by $25 \mathrm{AD}$ patients and 25 non-demented controls without neurological diseases or cognitive impairment, matched by age and gender to the study group. The number of patients was determined based on a previous pilot study (test power $=0.9$ ). We found a significant decrease in the activity of erythrocyte superoxide dismutase (SOD) and glutathione peroxidase (GPx), increased activity of catalase (CAT) and reduced concentration of plasma non-enzymatic antioxidants (uric acid, UA and reduced glutathione, GSH). In contrast, in the stimulated saliva of AD patients we observed significantly decreased activity of all antioxidant enzymes (SOD, CAT and GPx) as well as concentration of GSH compared to the control group. The content of lipid (malondialdehyde, MDA) and protein (advanced oxidation protein products, AOPP; advanced glycation end-products, AGE) oxidation products as well as biomarkers of nitrosative stress (peroxynitrite, nitrotyrosine) was significantly higher in both saliva and plasma of $A D$ patients compared to the controls. In AD patients, we also observed a considerable decrease in stimulated saliva secretion and salivary total protein content, and an increase in salivary $\beta$-amyloid concentration.

In conclusion, $A D$ results in redox imbalance towards oxidative reactions, both at the level of the oral cavity and the entire body. General redox balance disturbances do not coincide with salivary redox balance disturbances. Reduction in stimulated saliva secretion in AD patients reflects secretory dysfunction of the parotid glands.

\section{Introduction}

Neurodegenerative diseases are characterized by progressive degeneration of cells of the central and peripheral nervous system, which consequently leads to impairment of cognitive and motor functions. One of neurodegenerative diseases is Alzheimer's disease (AD) which, according to the available data, accounts for approximately $80 \%$ of all dementia cases ${ }^{1}$. AD is usually preceded by a period of mild cognitive impairment (MCl), i.e., short-term memory impairment, speech loss, being 'lost for words' as well as impaired orientation, concentration and attention. In the advanced stage of the disease, which usually increases with the patient's age, we can observe symptoms of depression, apathy, sleep disorders, delusions and hallucinations. For a long time, $\beta$-amyloid $(A \beta)$ deposits and neurofibrillary tangles were considered the main causative factors in the development of $A D$. Nowadays, it is known that the pathogenesis of this disease is more complex, and neuroinflammation and oxidative stress (OS) play a significant role in it. The latter phenomenon is described as imbalance between excessive formation of oxygen (ROS) and nitrogen (RNS) free radicals and their neutralization. Disruption of this delicate oxidoreductive balance results in oxidative modification/destruction of numerous cellular components, leading to cell/organ dysfunction and development of diseases. The brain is highly susceptible to redox imbalance due to its high-energy demand and high oxygen consumption in mitochondria, which makes them the main target of oxidative damage. However, it is also significant that $A \beta$ can build into the lipid bilayer of brain cells and enhance ROS production, and other important factors are the abundance of peroxidation-prone polyunsaturated fatty acids, high iron concentrations and a relatively poor panel of antioxidants in brain cells ${ }^{2}$.

Interestingly, numerous scientific publications indicate that patients with AD have systemic manifestations accompanying nervous system dysfunction, which suggests that the disease affects both the brain and the peripheral organs, including the salivary glands ${ }^{3,4}$.

In the salivary glands, not only has the presence of $A \beta$ deposits been confirmed, but also the expression of $A \beta$ and TAU protein in their acinar epithelial cells ${ }^{3,4}$. A significant increase in the salivary $A \beta_{1-42}$ in patients with mild and moderate $A D$ was also observed compared to patients with very advanced stage of the disease ${ }^{3}$, and significantly elevated t-TAU/p-TAU ratio was found in AD patients ${ }^{4}$. The results of Shi et al. ${ }^{4}$ suggest that salivary TAU can take a phosphorylated form which is essential for the development and progression of AD. In contrast to the results of Bermejo-Pareja et al. ${ }^{3}$, Kim et al.

5 observed positive correlation between salivary $A \beta$ and the stage of $A D$ progression.

Redox imbalance in the salivary glands and saliva of AD patients has not really been evaluated. However, it has been demonstrated that OS is a key factor in hyposalivation (reduced salivary secretion) in a group of patients with dementia of different etiology and varying degree of progression. Regardless of the type and severity of dementia (without confirmed AD), reduced antioxidant response of the salivary glands and increased modification of salivary cell elements were observed. It was noteworthy that reduced glutathione (GSH) deficiency is a factor differentiating patients with severe dementia from those with mild and moderate type of dementia 6,7 .

The aim of this study was to evaluate the antioxidant barrier and OS parameters in stimulated saliva and blood of patients with Alzheimer's disease.

\section{Materials And Methods}

\section{Patients}

The research was approved by the Bioethics Committee in Białystok, No. APK.002.95.2020. Patients qualified for the experiment had had the aim and methodology of the study explained. Informed consent was obtained from all participants. 10 patients with AD did not agree to have saliva and blood 
samples collected and they did not participate in the study. The study was performed in accordance with the Declaration of Helsinki and relevant local guidelines.

25 AD patients under observation in the Psychogeriatric Ward of SPP ZOZ (Independent Public Mental Health Care Centre) in Choroszcz were qualified for the study. The study also included 25 non-demented controls without neurological diseases or cognitive impairment, attending dental check-ups at the Department of Conservative Dentistry of the MUB (Medical University of Bialystok). The control group was matched to the study group in terms of gender and age. All AD patients were diagnosed by an experienced professor of psychiatry $(\mathrm{N}$. W.) and a neuropsychologist ( 0 . W.) according to the NINCDS-ADRDA criteria of $2007^{8}$. Establishing the diagnosis required finding cognitive decline (via a clinical mental examination) as well as impairment of social or occupational functions. The Mini-Mental State Examination (MMSE) was used to assess cognitive functions ${ }^{9}$. All patients in the study and control group had biochemical blood tests (vitamin B12, folic acid, thyroid function assessment, vitamin D3) performed. Patients in the study group also had brain imaging tests (brain MRI) done. However, 100\% confirmation of the disease is possible only after the death of the patient.

Exclusion criteria: history of hemorrhagic or ischemic stroke, traumas within the cerebral part of the skull, severe depression not allowing to establish the diagnosis of given dementia or determine its level, vascular dementia (based on DSM-III-R criteria / the FLAIR sequence of MRI confirming abnormalities in the medial temporal lobes, typical of inflammation or focal lesion of vascular origin), tumors within the brain, insulin resistance, diabetes, thyroid diseases, obesity, psoriasis, Parkinson's disease, Huntington's disease, deficiency of vitamin B12 or folic acid. Patients in the study and control groups did not abuse alcohol or smoke cigarettes. They had not taken antibiotics or supplements during the 6 months preceding material collection and had not changed their diet (AD patients - information obtained mostly from interviews with their family or hospital staff).

\section{Research Material}

The material for the study was collected between 6 and 7 a.m. between October and February, upon fasting and before oral hygiene procedures of the patients.

Due to the fact that it was impossible to collect unstimulated saliva (no saliva in the oral cavity), only stimulated whole saliva was used for the study. Saliva collection procedure was initiated in an environment familiar to the patient, usually at the bedside, after drinking a glass of water and a 5-minute conversation. Saliva was collected by an experienced and trained dentist (S. Z.). Patients had $100 \mu \mathrm{L}$ of citric acid sprayed on the tip of the tongue every 30 seconds for 10 minutes, and the saliva collected in the mouth was taken with a pipette (the procedure of spitting into a tube was not feasible in every case). The collected saliva was set aside in a container with ice and then centrifuged within 30 minutes from its collection ( $5000 \mathrm{~g}, 20 \mathrm{~min},+4^{\circ} \mathrm{C}$; MPW 351, MPW Med. Instruments, Warsaw, Poland). Salivary supernatant fluid enriched with butylated hydroxytoluene (BHT, $5 \mu \mathrm{L} 0.5 \mathrm{M} \mathrm{BHT}$ in acetonitrile per $0.5 \mathrm{~mL}$ of salivary supernatant) was retained for the study and stored at $-84^{\circ} \mathrm{C}$ for no longer than 6 months. The addition of $\mathrm{BHT}$ prevents oxidation of the sample ${ }^{10}$.

A dental examination was then performed by two experienced and previously calibrated dentists (A. K., A. Z). Objective symptoms of dry mouth ${ }^{11}$ and DMFT index ( $D$ - decayed, M - missing, F - filled, T - teeth) were assessed. If teeth were present, the gingival index (GI) was assessed as well ${ }^{12}$. Not all patients consented to the measurement of pocket depth; therefore, we refrained from this measurement.

Venous blood $(10 \mathrm{~mL})$ was collected by qualified hospital staff. Blood was collected using the S-Monovette® K3 EDTA blood collection system (Sarstedt, Nümbrecht, Germany). Within 30 minutes after collection, blood samples were centrifuged $\left(1500 \mathrm{~g}, 10 \mathrm{~min},+4^{\circ} \mathrm{C}\right.$; MPW 351 , MPW Med. Instruments, Warsaw, Poland). None of the samples underwent hemolysis. After separation of plasma, erythrocytes were rinsed three times with $0.9 \%$ cold $\mathrm{NaCl}$ solution and hemolyzed. BHT ( $5 \mu \mathrm{L} 0.5 \mathrm{M} \mathrm{BHT}$ in acetonitrile per $0.5 \mathrm{~mL}$ of sample) was added to the plasma and blood cell lysate. The samples were stored at $\sim 84^{\circ} \mathrm{C}$ for no longer than 6 months.

\section{Antioxidant Assays}

Superoxide dismutase (SOD, E.C. 1.15.1.1) activity was determined by measuring the rate of inhibition of adrenaline oxidation to adrenochrome using the spectrophotometric method at a wavelength of $480 \mathrm{~nm}$. It was assumed that one unit of SOD activity inhibits the oxidation of adrenaline by $50 \%{ }^{13}$.

The activity of catalase (CAT, E.C. 1.11.1.6) was measured spectrophotometrically at $240 \mathrm{~nm}$ wavelength based on the rate of hydrogen peroxide decomposition. One unit of CAT activity was defined as the amount of the enzyme that decomposes 1 mmol of hydrogen peroxide per one minute ${ }^{14}$.

Salivary peroxidase (Px, E.C. 1.11.1.7) activity was determined spectrophotometrically at $412 \mathrm{~nm}$ by measuring the decrease in the absorption of 5,5dithio-bis-(2-nitrobenzoic acid) (DTNB) to thionitrobenzoic acid. Measurements were made five times at intervals of 30 seconds. Erythrocyte glutathione peroxidase (GPx, EC 1.11.1.9) activity was assayed spectrophotometrically at $340 \mathrm{~nm}$ wavelength by determining the reduction of organic peroxides in the presence of NADPH. It was assumed that one unit of GPx catalyzes the process of oxidation of $1 \mu$ mol NADPH per one minute ${ }^{15}$.

Uric acid (UA) level was measured by the colorimetric method, using the commercial kit (QuantiChrom ${ }^{\text {TM }}$ Uric Acid DIUA-250; BioAssay Systems, Harward, CA, USA), as instructed by the manufacturer. In this method, 2,4,6-tripyridyl-s-triazine forms a blue complex with Fe ${ }^{3+}$ and UA. The absorbance was measured at $630 \mathrm{~nm}$ wavelength.

The concentration of reduced glutathione (GSH) was measured by the colorimetric method developed by Ellman with the use of DTNB. The absorbance was measured at $412 \mathrm{~nm}^{16}$. 


\section{Redox Status}

Total antioxidant capacity (TAC) was determined by the colorimetric method, which is based on the measurement of the ability to neutralize the radical cation $\mathrm{ABTS}^{\bullet+}$ [2,2-azino-bis- (3-ethylbenzothiazoline-6-sulfonate)] under the influence of antioxidants contained in the tested samples. The change in absorbance of the solution was measured at a wavelength of $660 \mathrm{~nm}^{17}$.

Total oxidation status (TOS) was assessed colorimetrically. This method is based on the oxidation of iron $\left(2^{+}\right)$ions to iron $\left(3^{+}\right)$ions in the presence of oxidants contained in the sample, followed by detection of $\mathrm{Fe}^{3+}$ ions by xylene orange. TOS was calculated from the standard curve for hydrogen peroxide and is reported in $\mathrm{nM}$ of $\mathrm{H}_{2} \mathrm{O}_{2}$ equivalent / $\mathrm{mg}$ of total proteins. TOS determination was performed in three samples ${ }^{18}$.

The oxidative stress index (OSI) was calculated according to the formula: OSI = TOS/TAS $\times 10019$

\section{Oxidative Damage Products}

Protein advanced glycation end products (AGE) concentration was estimated spectrofluorimetrically by measuring AGE-specific fluorescence at 350 $\mathrm{nm} / 440 \mathrm{~nm}$ wavelength. For AGE determination in plasma, samples were diluted 1:50 (v/v) in phosphate-buffered saline (PBS) ${ }^{20}$.

AOPP content was analyzed spectrophotometrically at $340 \mathrm{~nm}$ wavelength by measuring the oxidative capacity of iodine ion. For AOPP measured in plasma, samples were diluted 1:50 (v/v) in phosphate-buffered saline (PBS) ${ }^{21}$.

The concentration of malondialdehyde (MDA) was measured against blank sample as thiobarbituric acid reactive substances (TBARS), using a reference curve for 1,1,3,3-tetramethoxypropane.

Nitrosative Stress

Nitric oxide (NO) level was measured by the colorimetric method using sulfanilamide and N-(1-naphthyl)-ethylenediamine dihydrochloride. The absorbance of the obtained product was measured at $490 \mathrm{~nm}$ wavelength ${ }^{22,23}$.

Peroxynitrite level was measured colorimetrically based on peroxynitrite-mediated nitration leading to the formation of nitrophenol. The absorbance of the obtained complex was measured at 320 nm wavelength 24,25 .

Nitrotyrosine level was measured using the ELISA commercial kit (Nitrotyrosine ELISA; Immundiagnostik AG, Bensheim, Germany) according to the manufacturer's instructions.

\section{Amyloid Cross $\beta$-structure (A $\beta$ )}

To evaluate the $ß$-amyloid formation, $90 \mu \mathrm{L}$ of the analyzed samples was placed on the microplate and $10 \mu \mathrm{L}$ of Thioflavin T was added. The intensity of the amyloid associated with thioflavin T fluorescence was measured at 385/485 nm wavelength 26.

\section{Results}

\section{Clinical and Dental Characteristics}

There were no significant differences in biochemical parameters or blood morphology between patients with Alzheimer's disease and the control group. The flow of stimulated saliva as well as total protein concentration in SWS were significantly lower $(91 \% \downarrow p \leq 0.0001,42 \% \downarrow p=0.0022)$ in patients with $\mathrm{AD}$ compared to the controls. Clinical and dental data of Alzheimer's disease patients and the control group are presented in Table 1. 
Table 1

Stomatological and clinical characteristics of AD patients and the control group. AD - Alzheimer's disease; C - control group; SWS - stimulated whole saliva; DMFT - decayed, missing, filled teeth index; GI - gingival index; HGB - hemoglobin; HCT - hematocrit; CRP - C-reactive protein; AST aspartate transaminase; $A L T$ - alanine transferase; $\mathrm{Na}^{+}$- sodium; $\mathrm{K}^{+}$- potassium; TSH - thyroid stimulating hormone; Vit. $\mathrm{D}_{3}-$ vitamin $\mathrm{D}_{3} ;$ Vit. $\mathrm{B}_{12}-$ vitamin B12; MMSE - Mini Mental State Examination; CHD - coronary heart disease.

\begin{tabular}{|c|c|c|c|c|c|}
\hline & \multicolumn{2}{|l|}{ C } & \multicolumn{2}{|l|}{$A D$} & \multirow[t]{2}{*}{ P-value } \\
\hline & $\begin{array}{l}\text { Median (minimum - } \\
\text { maximum) }\end{array}$ & Mean \pm SD & $\begin{array}{l}\text { Median (minimum - } \\
\text { maximum) }\end{array}$ & Mean \pm SD & \\
\hline Age (years) & $83.6(66-90)$ & $82.1 \pm 6.67$ & $82.5(66-90)$ & $81.19 \pm 6.765$ & NS \\
\hline $\begin{array}{l}\text { Time from diagnosing the disease } \\
\text { (years) }\end{array}$ & - & - & $9(5-13)$ & $\begin{array}{l}9.043 \pm \\
2.30569\end{array}$ & - \\
\hline \multicolumn{6}{|l|}{ Stomatological characteristics } \\
\hline \multirow[t]{2}{*}{ SWS total protein $(\mu \mathrm{g} / \mathrm{mL})$} & 2314 & \multirow{2}{*}{$\begin{array}{l}2368 \pm \\
647.2\end{array}$} & 1338 & \multirow[t]{2}{*}{$1597 \pm 1065$} & \multirow[t]{2}{*}{0.0022} \\
\hline & $(1379-3872)$ & & $(203.8-4391)$ & & \\
\hline \multirow[t]{2}{*}{ SWS flow (mL/min) } & 1.465 & \multirow{2}{*}{$\begin{array}{l}1.364 \pm \\
0.479\end{array}$} & 0.12 & \multirow{2}{*}{$\begin{array}{l}0.09 \pm \\
0.06325\end{array}$} & \multirow{2}{*}{$\begin{array}{l}<.0001 \\
0.001\end{array}$} \\
\hline & $(0.5-2)$ & & $(0.09-0.26)$ & & \\
\hline DMFT & $28.44(25.44-29.98)$ & $\begin{array}{l}29.45 \pm \\
0.56\end{array}$ & $28.66(27.34-29.98)$ & $29.98 \pm 0.88$ & NS \\
\hline $\mathrm{GI}$ & $1.58(1.34-2.3)$ & $1.58 \pm 0.07$ & $1.99(1.59-2.18)$ & $2.05 \pm 0.15$ & NS \\
\hline \multicolumn{6}{|c|}{ Blood count and biochemical characteristics } \\
\hline Leukocytes $\left(10^{\wedge} 3 / \mu \mathrm{l}\right)$ & $7.87(3.55-9.7)$ & $\begin{array}{l}6.41 \pm \\
2.211\end{array}$ & $6.3(3.9-12.6)$ & $6.72 \pm 2.114$ & NS \\
\hline Erythrocytes $\left(10^{\wedge} 6 / \mu \mathrm{l}\right)$ & $4.21(3.92-5.61)$ & $\begin{array}{l}4.185 \pm \\
0.563\end{array}$ & $4.075(3.33-6.1)$ & $\begin{array}{l}4.233 \pm \\
0.6395\end{array}$ & NS \\
\hline \multirow[t]{2}{*}{$\mathrm{HGB}(\mathrm{g} / \mathrm{dL})$} & \multirow[t]{2}{*}{13.23 (11.9-17.42) } & \multirow{2}{*}{$\begin{array}{l}12.89 \pm \\
1.123\end{array}$} & 12.95 & \multirow[t]{2}{*}{$12.74 \pm 1.081$} & \multirow[t]{2}{*}{ NS } \\
\hline & & & $(9.7-14.2)$ & & \\
\hline \multirow[t]{2}{*}{ HCT (\%) } & \multirow[t]{2}{*}{$39.32(33.24-50.02)$} & \multirow{2}{*}{$\begin{array}{l}37.96 \pm \\
2.806\end{array}$} & 38.05 & \multirow[t]{2}{*}{$37.87 \pm 2.902$} & \multirow[t]{2}{*}{ NS } \\
\hline & & & $(29.3-42.3)$ & & \\
\hline \multirow[t]{2}{*}{ Glucose (mg/dL) } & \multirow[t]{2}{*}{$79(70-94)$} & \multirow{2}{*}{$\begin{array}{l}115.6 \pm \\
46.64\end{array}$} & 78 & \multirow[t]{2}{*}{$116.9 \pm 46.22$} & \multirow[t]{2}{*}{ NS } \\
\hline & & & $(68-96)$ & & \\
\hline $\mathrm{CRP}(\mathrm{mg} / \mathrm{L})$ & $3.2(0.2-12.45)$ & $23.8 \pm$ & 3.4 & $23.4 \pm 77.19$ & NS \\
\hline & & & $(0.1-37.1)$ & & \\
\hline AST (U/L) & $19(10-46)$ & $\begin{array}{l}25.89 \pm \\
12.42\end{array}$ & & $26.26 \pm 11.48$ & NS \\
\hline & & & $(12-63)$ & & \\
\hline ALT (U/L) & $14(7-55)$ & $\begin{array}{l}17.98 \pm \\
867\end{array}$ & 17 & $18.42 \pm 8.329$ & NS \\
\hline & & & $(7-35)$ & & \\
\hline $\mathrm{Na}^{+}(\mathrm{mmol} / \mathrm{L})$ & $143.6(136.5-140.2)$ & $\begin{array}{l}142.2 \pm \\
3321\end{array}$ & 142.3 & $141.5 \pm 2.922$ & NS \\
\hline & & & $(135.9-145.1)$ & & \\
\hline $\mathrm{K}^{+}(\mathrm{mmol} / \mathrm{L})$ & $4.58(3.87-5.8)$ & $9.87 \pm$ & 4.215 & $10.81 \pm 30.42$ & NS \\
\hline & & & $(3.71-147)$ & & \\
\hline TSH $(\mu \mathrm{IU} / \mathrm{mL})$ & $1.4(1.45-4.69)$ & $1.925 \pm$ & 1.34 & $1.857 \pm 1.201$ & NS \\
\hline & & & $(0.69-4.73)$ & & \\
\hline Vit. $D_{3}(n g / m L)$ & $20(12.13-53.23)$ & $18.67 \pm$ & 16.19 & $17.37 \pm 12.32$ & NS \\
\hline & & & $(3-43.67)$ & & \\
\hline Vit. $B_{12}(p g / m L)$ & $314.7(123-542)$ & $\begin{array}{l}256.6 \pm \\
132.5\end{array}$ & 214.3 & $222.7 \pm 121.2$ & NS \\
\hline & & & $(50-456.3)$ & & \\
\hline
\end{tabular}




\begin{tabular}{|c|c|c|c|c|c|}
\hline & \multicolumn{2}{|l|}{ c } & \multicolumn{2}{|l|}{$A D$} & \multirow[t]{2}{*}{ P-value } \\
\hline & $\begin{array}{l}\text { Median (minimum - } \\
\text { maximum) }\end{array}$ & Mean \pm SD & $\begin{array}{l}\text { Median (minimum - } \\
\text { maximum) }\end{array}$ & Mean \pm SD & \\
\hline \multirow[t]{2}{*}{ Folic acid $(\mathrm{ng} / \mathrm{mL})$} & \multirow[t]{2}{*}{$2.34(1.23-16.55)$} & \multirow{2}{*}{$\begin{array}{l}3.105 \pm \\
3.021\end{array}$} & 2.28 & \multirow[t]{2}{*}{$3.324 \pm 3.028$} & \multirow[t]{2}{*}{ NS } \\
\hline & & & $(0.88-12.53)$ & & \\
\hline \multicolumn{6}{|l|}{ Cognitive function } \\
\hline MMSE & $28(26-29)$ & $16 \pm 5.43$ & $15(0-20)$ & $14 \pm 4.69$ & NS \\
\hline \multicolumn{6}{|l|}{ Concomitant diseases } \\
\hline Hypertension n (\%) & $14(56)$ & & $16(64)$ & & NS \\
\hline Diabetes $\mathrm{n}(\%)$ & $2(8)$ & & $3(12)$ & & NS \\
\hline CHD n (\%) & $5(20)$ & & $6(24)$ & & NS \\
\hline Atherosclerosis n (\%) & $3(12)$ & & $3(12)$ & & NS \\
\hline Osteoporosis n (\%) & $1(4)$ & & $2(8)$ & & NS \\
\hline
\end{tabular}

In the $\mathrm{AD}$ group, significantly poorer hydration of the vermilion zone and buccal mucosa was observed. The condition of tongue mucosa was also worse compared to the control group (Table 2).

Table 2

Assessment of dryness of the oral cavity in $A D$ patients and healthy controls. $A D$

\begin{tabular}{|c|c|c|c|}
\hline Assessment n (\%) & $A D$ & C & $\mathbf{p}$ \\
\hline \multicolumn{4}{|c|}{ of vermilion zone hydration } \\
\hline 0 & 0 & $3(12 \%)$ & $<0.0001$ \\
\hline 1 & $1(4 \%)$ & $18(72 \%)$ & \\
\hline 2 & $2(8 \%)$ & $2(8 \%)$ & \\
\hline 3 & $22(88 \%)$ & $2(8 \%)$ & \\
\hline \multicolumn{4}{|c|}{ of buccal mucosa hydration } \\
\hline 1 & 0 & $15(60 \%)$ & $<0.0001$ \\
\hline 2 & 0 & $10(40 \%)$ & \\
\hline 3 & $25(100 \%)$ & 0 & \\
\hline \multicolumn{4}{|c|}{$\begin{array}{l}\text { of the condition of tongue } \\
\text { mucosa }\end{array}$} \\
\hline 1 & 0 & $23(92 \%)$ & $<0.0001$ \\
\hline 2 & $25(100 \%)$ & $2(8 \%)$ & \\
\hline \multicolumn{4}{|c|}{ palpation of salivary glands } \\
\hline 1 & $1(4 \%)$ & $25(100 \%)$ & $<0.0001$ \\
\hline 2 & $24(96 \%)$ & 0 & \\
\hline
\end{tabular}

\section{Redox Homeostasis}

The results of redox balance parameters in saliva, erythrocytes and plasma of AD patients and controls are presented in Table 3 . 
Table 3

Salivary and erythrocytes/plasma redox biomarkers in patients with Alzheimer's disease and the control group. C - control group; AD - Alzheimer's disease; SOD - superoxide dismutase; CAT - catalase; GPx - glutathione peroxidase; UA - uric acid; GSH - glutathione; TAC - mean total antioxidant capacity; TOS - mean total oxidant status; OSI - oxidative stress index; AGE - advanced glycation end products; AOPP - advanced oxidation protein products; MDA - malondialdehyde; NO - nitric oxide, A $\beta$ - amyloid beta.

\begin{tabular}{|c|c|c|c|c|c|c|c|c|c|c|}
\hline \multirow[t]{3}{*}{ Biomarker } & \multicolumn{5}{|l|}{ Saliva } & \multicolumn{5}{|c|}{ Erythrocytes/Plasma } \\
\hline & \multicolumn{2}{|l|}{ C } & \multicolumn{2}{|l|}{$A D$} & \multirow[t]{2}{*}{ P-value } & \multicolumn{2}{|l|}{ C } & \multicolumn{2}{|l|}{$A D$} & \multirow[t]{2}{*}{ P-value } \\
\hline & $\begin{array}{l}\text { Median } \\
\text { (minimum } \\
- \\
\text { maximum) }\end{array}$ & $\begin{array}{l}25 \%- \\
75 \% \\
\text { Percentile }\end{array}$ & $\begin{array}{l}\text { Median } \\
\text { (minimum } \\
- \\
\text { maximum) }\end{array}$ & $\begin{array}{l}25 \%- \\
75 \% \\
\text { Percentile }\end{array}$ & & $\begin{array}{l}\text { Median } \\
\text { (minimum } \\
- \\
\text { maximum) }\end{array}$ & $\begin{array}{l}25 \%- \\
75 \% \\
\text { Percentile }\end{array}$ & $\begin{array}{l}\text { Median } \\
\text { (minimum } \\
- \\
\text { maximum) }\end{array}$ & $\begin{array}{l}25 \%- \\
75 \% \\
\text { Percentile }\end{array}$ & \\
\hline \multicolumn{11}{|c|}{ Antioxidant barrier } \\
\hline \multirow{2}{*}{$\begin{array}{l}\text { SOD } \\
\text { (mU/mg } \\
\text { protein) }\end{array}$} & 1.142 & \multirow{2}{*}{$\begin{array}{l}0.9785- \\
1.186\end{array}$} & 0.6669 & \multirow{2}{*}{$\begin{array}{l}0.3298- \\
1.1\end{array}$} & \multirow[t]{2}{*}{0.0007} & 0.6002 & \multirow{2}{*}{$\begin{array}{l}0.5029- \\
0.7711\end{array}$} & 0.5019 & \multirow{2}{*}{$\begin{array}{l}0.08679 \\
-0.6873\end{array}$} & \multirow[t]{2}{*}{0.0092} \\
\hline & $\begin{array}{l}(0.8383- \\
1.239)\end{array}$ & & $\begin{array}{l}(0.05171- \\
1.271)\end{array}$ & & & $\begin{array}{l}(0.1618- \\
1.156)\end{array}$ & & $\begin{array}{l}(0.003865- \\
0.9597)\end{array}$ & & \\
\hline \multirow{2}{*}{$\begin{array}{l}\mathrm{CAT} \text { (nmol } \\
\mathrm{H}_{2} \mathrm{O}_{2} / \mathrm{min} / \mathrm{mg} \\
\text { protein) }\end{array}$} & 0.5761 & \multirow{2}{*}{$\begin{array}{l}0.4879- \\
0.67\end{array}$} & 0.1197 & \multirow{2}{*}{$\begin{array}{l}0.065- \\
0.3442\end{array}$} & \multirow[t]{2}{*}{$<0.0001$} & 0.1721 & \multirow{2}{*}{$\begin{array}{l}0.1539- \\
0.1914\end{array}$} & 0.2361 & \multirow{2}{*}{$\begin{array}{l}0.1239- \\
0.9597\end{array}$} & \multirow[t]{2}{*}{0.0222} \\
\hline & $\begin{array}{l}(0.2327- \\
0.7137)\end{array}$ & & $\begin{array}{l}(0.001944 \\
-0.8383)\end{array}$ & & & $\begin{array}{l}(0.1353- \\
0.2203)\end{array}$ & & $\begin{array}{l}(0.02056- \\
0.9597)\end{array}$ & & \\
\hline \multirow{3}{*}{$\begin{array}{l}\mathrm{GPx}(\mathrm{mU} / \mathrm{mg} \\
\text { protein) }\end{array}$} & 0.07983 & & 0.06871 & 0.06453 & 0.0037 & 0.1345 & & & 0.05625 & $<0.0001$ \\
\hline & $\begin{array}{l}(0.063- \\
0.094)\end{array}$ & \multirow[t]{2}{*}{$\begin{array}{l}0.07676 \\
-0.0875\end{array}$} & \multirow[t]{2}{*}{$\begin{array}{l}(0.06084- \\
0.151)\end{array}$} & \multirow[t]{2}{*}{0.07975} & & $\begin{array}{l}(0.1026- \\
0.1503)\end{array}$ & $\begin{array}{l}0.1265- \\
0.1419\end{array}$ & & & \\
\hline & & & & & & & & $\begin{array}{l}(0.04046- \\
0.1628)\end{array}$ & & \\
\hline $\mathrm{UA}(\mu \mathrm{g} / \mathrm{mg}$ & 67.28 & $\begin{array}{l}61.01 \text { - } \\
75.6\end{array}$ & 60.55 & $\begin{array}{l}26.17- \\
84.3\end{array}$ & 0.3895 & 29.28 & 28.18 - & 40.54 & $35.08-$ & $<0.0001$ \\
\hline & $\begin{array}{l}(50.6- \\
85.52)\end{array}$ & & $\begin{array}{l}(25.46- \\
113)\end{array}$ & & & $\begin{array}{l}(24.71- \\
40.9)\end{array}$ & & $\begin{array}{l}(11.67- \\
57.98)\end{array}$ & & \\
\hline GSH (ng/mg & 2.361 & $2.14-$ & 2.103 & $2.024-$ & 0.0312 & 2.808 & $2.342-$ & 2.162 & $1.589-$ & $<0.0001$ \\
\hline & $\begin{array}{l}(1.293- \\
2.951)\end{array}$ & & $\begin{array}{l}(1.265- \\
2.335)\end{array}$ & & & $\begin{array}{l}(1.52- \\
3.272)\end{array}$ & & $\begin{array}{l}(0.6856- \\
2.776)\end{array}$ & & \\
\hline Redox status & & & & & & & & & & \\
\hline $\begin{array}{l}\text { TAC } \\
\text { (umol/mg }\end{array}$ & & $\begin{array}{l}4.931- \\
6.592\end{array}$ & & $\begin{array}{l}5.825- \\
6253\end{array}$ & 0.838 & & $\begin{array}{l}7.635 \text { - } \\
1034\end{array}$ & & $5.46-$ & $<0.0001$ \\
\hline protein) & $\begin{array}{l}(4.031- \\
7.127)\end{array}$ & & $\begin{array}{l}(5.351- \\
7.074)\end{array}$ & & & $\begin{array}{l}(4.366- \\
12.18)\end{array}$ & & $\begin{array}{l}(4.37- \\
6.833)\end{array}$ & & \\
\hline TOS & 197.5 & 192.5 - & 392.5 & $\begin{array}{l}385.5- \\
3964\end{array}$ & $<0.0001$ & 209.5 & $\begin{array}{l}189.2 \text { - } \\
231.4\end{array}$ & 410.3 & $\begin{array}{l}391.6- \\
4502\end{array}$ & $<0.0001$ \\
\hline protein) & $\begin{array}{l}(176.7- \\
496.8)\end{array}$ & & $\begin{array}{l}(353.9- \\
423.2)\end{array}$ & & & $\begin{array}{l}(73.17- \\
383)\end{array}$ & & $\begin{array}{l}(361.8- \\
584.6)\end{array}$ & & \\
\hline $\begin{array}{l}\text { OSI } \\
\text { (TOS/TAC }\end{array}$ & 33.62 & $\begin{array}{l}29.55- \\
43.43\end{array}$ & 64.53 & $\begin{array}{l}61.82- \\
68.62\end{array}$ & $<0.0001$ & 22.26 & $\begin{array}{l}18.56- \\
285\end{array}$ & 72.98 & $\begin{array}{l}67.08- \\
8427\end{array}$ & $<0.0001$ \\
\hline ratio) & $\begin{array}{l}(26.74- \\
78.92)\end{array}$ & & $\begin{array}{l}(55.77- \\
72.84)\end{array}$ & & & $\begin{array}{l}(6.656- \\
80.68)\end{array}$ & & $\begin{array}{l}(58.12- \\
100.7)\end{array}$ & & \\
\hline Oxidative and $n$ & rosative stres & & & & & & & & & \\
\hline AGE & 0.8543 & 0.6636 - & 1.055 & 1.018 - & $<0.0001$ & 1.255 & 1.119 - & 1.847 & $1.628-$ & $<0.0001$ \\
\hline $\begin{array}{l}\text { (AFU/mg } \\
\text { protein) }\end{array}$ & $\begin{array}{l}(0.4644- \\
0.9969)\end{array}$ & & $\begin{array}{l}(0.5435- \\
1.592)\end{array}$ & & & $\begin{array}{l}(1.017- \\
1.313)\end{array}$ & & $\begin{array}{l}(0.621- \\
3.494)\end{array}$ & & \\
\hline AOPP & 19.65 & $\begin{array}{l}9.784- \\
23.8\end{array}$ & & $\begin{array}{l}17.65- \\
27.77\end{array}$ & 0.0285 & & $\begin{array}{l}5.116- \\
7.396\end{array}$ & & $\begin{array}{l}8.074- \\
10.56\end{array}$ & $<0.0001$ \\
\hline $\begin{array}{l}\text { (nmol/mg } \\
\text { protein) }\end{array}$ & $\begin{array}{l}(4.231- \\
27.45)\end{array}$ & & $\begin{array}{l}(13.13- \\
49.84)\end{array}$ & & & $\begin{array}{l}(2.14- \\
8.096)\end{array}$ & & $\begin{array}{l}(6.573- \\
11.06)\end{array}$ & & \\
\hline MDA & 207.3 & $\begin{array}{l}159.9- \\
402.8\end{array}$ & 582.2 & $\begin{array}{l}138.3- \\
998.3\end{array}$ & 0.0297 & & $\begin{array}{l}261.7- \\
415.1\end{array}$ & & $\begin{array}{l}372.3- \\
489.4\end{array}$ & 0.0004 \\
\hline $\begin{array}{l}(\mu \mathrm{g} / \mathrm{mg} \\
\text { protein) }\end{array}$ & $\begin{array}{l}(119.2- \\
402.8)\end{array}$ & & $\begin{array}{l}\text { (61.29- } \\
1957)\end{array}$ & & & $\begin{array}{l}(186.1- \\
486.4)\end{array}$ & & $(361-1281)$ & & \\
\hline $\begin{array}{l}\text { NO (ng/mg } \\
\text { protein) }\end{array}$ & 0.183 & & 0.223 & $\begin{array}{l}0.153- \\
0.3111\end{array}$ & 0.0371 & & $\begin{array}{l}0.07392 \\
-0.1453\end{array}$ & 0.1582 & $\begin{array}{l}0.08975 \\
-02918\end{array}$ & 0.0669 \\
\hline & $\begin{array}{l}(0.05893- \\
0.3237)\end{array}$ & $\begin{array}{l}0.1003- \\
0.2318\end{array}$ & $\begin{array}{l}(0.1174- \\
0.5003)\end{array}$ & & & $\begin{array}{l}(0.01694- \\
0.2289)\end{array}$ & & $\begin{array}{l}(0.0555- \\
0.521)\end{array}$ & & \\
\hline Peroxynitrite & 15.36 & & 17.75 & $\begin{array}{l}16.27- \\
19.99\end{array}$ & 0.0001 & 205.9 & $\begin{array}{l}172.3- \\
251.1\end{array}$ & 215.6 & $\begin{array}{l}198.8- \\
331.8\end{array}$ & \\
\hline
\end{tabular}




\begin{tabular}{|c|c|c|c|c|c|c|c|c|c|c|}
\hline $\begin{array}{l}\text { (nmol/mg } \\
\text { protein) }\end{array}$ & $\begin{array}{l}(12.78- \\
20.95)\end{array}$ & $\begin{array}{l}14.59- \\
16.57\end{array}$ & $\begin{array}{l}(15.05- \\
57.14)\end{array}$ & & & $\begin{array}{l}(139.6- \\
318.7)\end{array}$ & & $\begin{array}{l}(191- \\
331.8)\end{array}$ & & 0.0344 \\
\hline $\begin{array}{l}\text { Nitrotyrosine } \\
\text { (pmol/mg } \\
\text { protein) }\end{array}$ & $\begin{array}{l}1995 \\
(1693- \\
2382)\end{array}$ & $\begin{array}{l}1883- \\
2250\end{array}$ & $\begin{array}{l}2322 \\
(1182- \\
3969)\end{array}$ & $\begin{array}{l}1959- \\
2889\end{array}$ & 0.0175 & $\begin{array}{l}2923 \\
(2368- \\
3421)\end{array}$ & $\begin{array}{l}2694- \\
3311\end{array}$ & $\begin{array}{l}5675 \\
\\
(1061- \\
9402)\end{array}$ & $\begin{array}{l}4513- \\
7405\end{array}$ & $<0.0001$ \\
\hline \multicolumn{11}{|l|}{ Amyloid $\beta$} \\
\hline $\begin{array}{l}\mathrm{A} \beta \text { (AFU/mg } \\
\text { protein) }\end{array}$ & $\begin{array}{l}5049 \\
(678.7- \\
6512)\end{array}$ & $\begin{array}{l}3691- \\
5476\end{array}$ & $\begin{array}{l}7236 \\
(5402- \\
18396)\end{array}$ & $\begin{array}{l}6351- \\
9299\end{array}$ & $<0.0001$ & $\begin{array}{l}70487 \\
(26787- \\
85117)\end{array}$ & $\begin{array}{l}56906- \\
74762\end{array}$ & $\begin{array}{l}70948 \\
(33935- \\
102063)\end{array}$ & $\begin{array}{l}60802- \\
78992\end{array}$ & 0.5405 \\
\hline
\end{tabular}

\section{Antioxidants}

The activity of SOD, CAT and GPx in SWS of AD patients was significantly lower compared to the control group ( $\downarrow 42 \% p=0.0007, \downarrow 79 \% p \leq 0.0001$, $\downarrow 15 \% p=0.0037$, respectively). Similarly, the concentration of GSH in the SWS of patients with Alzheimer's disease was considerably lower ( $\downarrow 11 \% \mathrm{p}=$ 0.0312) compared to the control group. The concentration of UA in the SWS of AD patients as compared to the control group was at a similar level, not reaching statistical significance.

On the other hand, significantly lower SOD and GPx activity and GSH levels $(\downarrow 17 \% p=0.0092, \downarrow 36 \% p \leq 0.0001, \downarrow 23 \% p \leq 0.0001$, respectively) were observed in erythrocytes/plasma of the participants from the study group. CAT erythrocyte activity and concentration of plasma UA were significantly higher $(\uparrow 27 \% p=0.0222, \uparrow 28 \% p \leq 0.0001$, respectively) in the group of patients with Alzheimer's disease compared to the control group.

\section{Redox Status}

In SWS of patients with Alzheimer's disease, TOS as well as OSI were considerably higher ( $\uparrow 50 \% p \leq 0.0001,48 \% p \leq 0.0001$, respectively) than in the control group. At the same time, there was no significant difference in the level of salivary TAC between those two groups. The plasma of patients with Alzheimer's disease was characterized by a significantly lower level of TAC ( $\downarrow 37 \%$ p $\leq 0.0001)$ with a considerably higher levels of TOS and OSI ( $\uparrow 49 \%$ $p \leq 0.0001, \uparrow 69 \% p \leq 0.0001)$ compared to the control group.

\section{Oxidative Damage Products}

The group of patients with Alzheimer's disease was characterized by a considerably higher concentrations of AGE, AOPP and MDA ( $\uparrow 19 \% \mathrm{p} \leq 0.0001$, $\uparrow 12 \% p=0.0285, \uparrow 64 \% p=0.0297$, respectively) in SWS compared to the control group. Similar results were observed in the plasma of the study group, where the concentrations of AGE, AOPP, MDA were also considerably higher ( $\uparrow 32 \% p \leq 0.0001, \uparrow 30 \% p \leq 0.0001, \uparrow 21 \% p=0.0004$, respectively)

compared to the controls.

\section{Nitrosative Stress}

The concentrations of NO, peroxynitrile and nitrotyrosine in SWS of AD patients were significantly higher than in the control group $(\uparrow 18 \% \mathrm{p}=0.0371$, $\uparrow 13 \% p=0.0001, \uparrow 14 \% p=0.0175$, respectively). Similar results were obtained in the plasma of patients from the study group where the concentrations of NO, peroxynitrile and nitrotyrosine were considerably higher than in the control group $(\uparrow 33 \% p=0.0669, \uparrow 5 \% p=0.0344, \uparrow 48 \% p \leq 0.0001$, respectively).

\section{Amyloid Cross $\beta$-structure}

The concentration of A $\beta$ in SWS of patients with Alzheimer's disease was significantly higher ( $\uparrow 30 \% p \leq 0.0001)$ compared to the control group. In the plasma, there was no significant difference in $A \beta$ concentration between those two groups.

\section{Correlations}

Correlations between salivary redox biomarkers and activity of the salivary glands as well as AD severity are presented in Table 4. It is particularly noteworthy that we found a statistically significant positive correlation between FR and MMSE and a negative correlation between FR and AOPP in the SWS of AD patients. Within the scope of assays including salivary antioxidants in the SWS of patients with AD, we observed positive correlations between Px as well as SOD and time elapsed from AD diagnosis, and A level and SOD activity. Additionally, the concentration of AOPP in SWS of the study group correlated positively with buccal mucosa hydration and negatively with GSH. 
Table 4

Statistically significant correlations between redox biomarkers and clinical parameters. AD - Alzheimer's disease; MMSE - Mini-Mental State Examination; SWS - stimulated whole saliva; MDA malondialdehyde; AOPP - advanced oxidation protein products; GSH reduced glutathione; GPx - glutathione peroxidase; SOD - superoxide dismutase; $A \beta-$ amyloid $\beta ; C$ - control group

\begin{tabular}{|lll|}
\hline Pair of variables & $\mathbf{r}$ & $\mathbf{p}$ \\
\hline AD & & \\
\hline MMSE and SWS flow & 0.908 & $<0.0001$ \\
\hline MMSE and plasma MDA & 0.955 & $<0.0001$ \\
\hline SWS AOPP and SWS flow & -0.672 & 0.002 \\
\hline SWS AOPP and SWS GSH & -0.607 & 0.003 \\
\hline SWS Px and time elapsed from the diagnosis & 0.877 & $<0.0001$ \\
\hline SWS SOD and time elapsed from the diagnosis & 0.677 & 0.004 \\
\hline SWS SOD and SWS A & 0.732 & 0.0001 \\
\hline C & & \\
\hline AOPP and hydration of buccal mucosa & 0.747 & $<0.0001$ \\
\hline
\end{tabular}

\section{Discussion}

The main results of the presented study indicate that although salivary total antioxidant status (TAC) in AD patients was unaltered, we observed increased oxidative modification of cellular elements of the salivary glands and structures present in the oral cavity. Saliva is not only the secretion of the salivary glands, but it also contains exfoliated oral mucosal epithelium and gingival fluid reflecting the redox status of periodontal tissues. The finding that unstimulated salivary flow from the submandibular glands is impaired in AD patients has already been published ${ }^{27}$. However, it can be concluded that in the AD patients from our experiment the submandibular glands were completely dysfunctional. Despite using several methods of collecting unstimulated saliva, we were unable to collect the material. Even gentle attempts to collect saliva (using a pipette) from the mucous membranes of the lips, palate and cheeks failed. Moreover, we demonstrated high secretory failure of the parotid glands that secrete stimulated saliva. Despite providing hydration of the patient's oral cavity (each patient drank a glass of water, and nurses reported that patients regularly drink various types of liquids) and collecting the material in autumn and winter (at room temperature of $20-21^{\circ} \mathrm{C}$ ), secretion of stimulated saliva in $A D$ patients was reduced compared to their peers of the control group, as normal stimulated saliva secretion starts with the value of $0.7 \mathrm{~mL} / \mathrm{min}$. Stimulated secretion was at the level of $0.12 \mathrm{~mL} / \mathrm{min}$, which indicates severe hyposalivation. The cause of reduced salivary secretion in AD patients is unknown. Saliva secretion is initiated by reflex-induced nerve impulses. Controlling this process depends on neurotransmitters released at nerve ends in the salivary glands. Typical neurotransmitters associated with water secretion are acetylcholine (ACh) and numerous neuropeptide neurotransmitters ${ }^{28}$. In the brain of AD patients we observed increased activity of acetylcholinesterase, an enzyme which breaks down ACh, followed by decreased ACh levels ${ }^{29}$. This deficit in the cholinergic function was connected with loss of memory as well as cognitive and learning ability in AD individuals ${ }^{29}$. Sayer et al. ${ }^{30}$ reported decreased salivary concentration of ACh in AD patients who did not respond to AChE-I treatment. The positive correlation between SWS and Mini-Mental State Examination may prove that declined cholinergic conductivity could be a cause of hyposalivation. The negative correlation between AOPP levels and SWS may also be considered as some kind of explanation. It was demonstrated that protein oxidation can accelerate the formation of toxic protein aggregates in the nucleus and cytoplasm of the nerves ${ }^{31}$. This may inhibit neurotransmitter release or reduce salivary gland innervation and thus decrease secretory response of the salivary glands. Decreased protein levels in the SWS of patients with AD vs healthy controls may evidence reduced activity of the sympathetic nervous system, whose stimulation determines protein synthesis in the salivary glands. Due to the selection of the control group (age of patients), we excluded age-related salivary gland changes (fatty and fibrous degeneration) as a cause of salivary gland dysfunction. With age, the number of medications taken increases, and many of them may affect saliva secretion and composition. Patients in both study groups had similar general diseases (hypertension, diabetes, coronary heart disease, atherosclerosis, osteoporosis) and were taking similar groups of drugs, so connecting hyposalivation with a particular disease or drug-related condition would be an oversimplification. With such significant deficiency of saliva, it is not surprising to find poor hydration of the vermilion zone, buccal mucosa or tongue condition. The positive correlation between buccal mucosal hydration and AOPP concentration in the control group may result from increased oxidative modification of moisturizing proteins, i.e., mucins and other glycoproteins, accompanying the older age, which entails changes in the coating properties of saliva. Assessment of xerostomia (subjective perception of dryness in the oral cavity) was not possible in every case; therefore, we refrained from presentation of the results.

Analysis of the results obtained in plasma and red cell lysate revealed a significant decrease in SOD and GPx activity ( $\exists 17 \%, \exists 39 \%$, respectively), increase in CAT activity (\#17\%), decrease in the concentration of non-enzymatic antioxidants ( $\exists 38 \%$ UA, $\exists 25 \%$ GSH, $\exists 39 \%$ TAC) and the existence of general OS (\#95\% TOS, \#227\% OSI, \#26\% MDA, \#47\% AGE, \#42\% AOPP) and nitrosative stress (\#4\% peroxynitrite, \#94\% nitrotyrosine). TAC is the resultant capacity of a given biological material to counteract specific oxidation reactions ${ }^{32}$. It is believed that TAC level corresponds to the antioxidant 
capacity of non-enzymatic antioxidants in the sample. TOS reflects the level of all free radicals and non-free radical molecules. OSI is the so-called oxidative stress index, that is the ratio of antioxidants to oxidants present in the assayed material ${ }^{33}$.

Changes in the redox balance in SWS are shifted towards oxidative processes similarly to plasma/blood cells, although not exactly to the same extent. In stimulated saliva of AD patients, we observed significantly decreased activity of all antioxidant enzymes: SOD, CAT and GPx ( $\exists 42 \%, \exists 80 \%, \exists 15 \%$, respectively) compared to the control group. Decreased parameters of the these components of the antioxidant barrier may result mainly from highly increased production of ROS (98\% \#TOS) which consistently utilize antioxidants in their combating. It may also be due to oxidative modifications of protein chains of the mentioned enzymes (12\% \#AOPP, $20 \%$ \#AGE), leading to their inactivation, as observed in the brain of AD patients ${ }^{34}$. Decreased GPx activity demonstrates antioxidant failure as well as impairment of other functions (not related to redox balance) of the parotid glands. As the only type of proteins studied, GPx is synthesized exclusively in the acinar cells of the salivary glands and is recognized as a marker of proper function of the parotid glands ${ }^{35,36}$. Interestingly, reduction of SOD and GPx activity in the SWS of AD patients correlated positively with the time elapsed from AD diagnosis; in addition, decreased SOD activity correlated positively with A levels in the SWS. The latter correlation may be caused by high affinity of $A \beta$ to bind $\mathrm{Cu}^{2+}$, which deprives saliva of this trace element ${ }^{37} \cdot \mathrm{Cu}^{2+}$ is a cofactor of the dismutation reaction catalyzed by SOD ${ }^{38}$.

GSH deficiency $(\exists 11 \%)$ accompanying negative correlation between GSH and AOPP may be due to increased oxidation of proteins that form oral cavity structures. Indeed, the main function of GSH is to maintain a reduced state of the thiol groups of proteins. The reduction of GSH may also result from deficiency of substrates involved in the regeneration of GSH. Another cause may be oxidative damage to GSH reductase, an enzyme that catalyzes the conversion of GSSG (oxidized form of glutathione) to GSH, with NADPH as the reducing cofactor ${ }^{39}$. A decrease in NADPH generation observed in the brain of $A D$ patients ${ }^{40}$ could hinder the re-synthesis of salivary GSH. Reduction in GSH concentration in the saliva may enhance $\mathrm{OH}$. formation, thus increasing the ROS load and be the cause of the increase in the reported concentrations of peroxynitrite (\#15\%) and nitrotyrosine (\#16\%) in the SWS of $A D$ patients. GSH is known as the more potent detoxification agent of peroxynitrite ${ }^{39}$. It was evidenced that peroxynitrite is involved in the pathogenesis of $A D$. It was observed that peroxynitrite simultaneously induces hyperphosphorylation, nitration and accumulation of tau protein ${ }^{41}$. The thus modified tau protein (p-tau) aggregates to form intracellular neurofibrillary tangles, exerting a toxic effect ${ }^{41}$. It is noteworthy that there was greater reduction in plasma GSH level $(\exists 25 \%)$ than in the SWS level $(\exists 11 \%)$ in AD patients, with simultaneous greater increase in plasma nitrosative stress expressed by nitrotyrosine levels (\#94\% vs \#16\%).

Only UA and TAC in stimulated saliva of AD patients did not differ compared to the controls. Despite unchanged TAC levels, salivary antioxidant systems were unable to counterbalance the increased ROS/RNS formation (\#TOS) and prevent the development of OS (\#91\% OSI, \#AOPP, \#AGE, \#180\% MDA). The reason for the accumulation of products of cellular elements oxidation/peroxidation could undoubtedly be malfunctioning of the repair systems responsible for the removal of defective macromolecules, which is typical in the course of AD ${ }^{42}$. Increased MDA concentration in SWS suggests the existence of advanced stages of free radical processes in the cells of salivary glands as well as oral cavity structures of $A D$ patients compared to the control group ${ }^{43}$, as membrane lipid molecules are thought to undergo oxidative modification with generation of reactive aldehydes at higher concentrations of ROS than proteins ${ }^{44}$. Interestingly, we demonstrated a positive correlation between serum MDA concentration and MMSE. It can be assumed that the level of MDA reflects the degree of peroxidation of unsaturated fatty acids of the biological membranes and thus changes of membrane fluidity. Membrane fluidity determines the normal functioning of cells, including neurons, so an increase in serum MDA content could be linked to neuronal loss in AD.

A weakness of the presented study was its small panel of parameters examined, which was determined by the very small amount of saliva available for assays.

\section{Conclusions}

1. Alzheimer's disease results in redox imbalance towards oxidation reactions, both at the level of the oral cavity and the entire body.

2. General redox balance disturbances do not coincide with salivary redox balance disturbances, which means that both processes occur independently.

3. Reduction in the activity of key antioxidant enzymes in SWS correlates with the time elapsed from the AD diagnosis.

4. Reduction in stimulated saliva secretion in AD patients reflects secretory failure of the parotid glands. The function of the submandibular salivary glands responsible for unstimulated saliva secretion is completely impaired in the course of the disease.

5. Secretory failure of the parotid glands may be associated with declined cholinergic conductivity; however, it could also depend on oxidative modification of proteins.

\section{Declarations}

\section{Authors contributions:}

Conceptualization, A.Z., M.M., Data curation, A.Z. and M.M.; S.Z., , Formal analysis, A.Z. and I.Sz.; Funding acquisition, A.Z.; S.Z. Investigation, A.Z. A.K., S.Z., N.W., O.W., R.M., and M.M.; Methodology, A.Z., M.M., S.Z., A.K..; Material collection: A.Z., S.Z., A.K.; Supervision, A.Z., M.M., D.K., N.W; Validation, A.Z. and M.M.; Visualization, A.Z. and M.M.; Writing-original draft, A.Z., A.K., S.Z., M.M.; Writing-review and editing, A.Z. and M.M. 


\section{Funding:}

This work was granted by the Medical University of Bialystok, Poland (grant number: SUB/1/DN/21/002/3330), SUB/1/DN/21/001/1209.

\section{Data Availability Statement:}

The datasets generated for this study are available on request to the corresponding author.

\section{References}

1. Pawlik, P. \& Błochowiak, K. The Role of Salivary Biomarkers in the Early Diagnosis of Alzheimer's Disease and Parkinson's Disease. Diagnostics (Basel) 11, 371, doi:10.3390/diagnostics11020371 (2021).

2. Ansari, M. A. \& Scheff, S. W. Oxidative stress in the progression of Alzheimer disease in the frontal cortex. J Neuropathol Exp Neurol 69, 155-167, doi:10.1097/NEN.0b013e3181cb5af4 (2010).

3. Bermejo-Pareja, F., Antequera, D., Vargas, T., Molina, J. A. \& Carro, E. Saliva levels of Abeta1-42 as potential biomarker of Alzheimer's disease: a pilot study. BMC Neuro/ 10, 108, doi:10.1186/1471-2377-10-108 (2010).

4. Shi, M. et al. Salivary tau species are potential biomarkers of Alzheimer's disease. J Alzheimers Dis 27, 299-305, doi:10.3233/jad-2011-110731 (2011)

5. Kim, C. B., Choi, Y. Y., Song, W. K. \& Song, K. B. Antibody-based magnetic nanoparticle immunoassay for quantification of Alzheimer's disease pathogenic factor. J Biomed Opt 19, 051205, doi:10.1117/1.Jbo.19.5.051205 (2014).

6. Choromańska, M. et al. Antioxidant defence, oxidative stress and oxidative damage in saliva, plasma and erythrocytes of dementia patients. Can salivary AGE be a marker of dementia? Int J Mol Sci 18, 2205, doi:10.3390/ijms18102205 (2017).

7. Klimiuk, A. et al. Salivary Redox Biomarkers in Different Stages of Dementia Severity. Journal of clinical medicine 8, 840, doi:10.3390/jcm8060840 (2019)

8. Dubois, B. et al. Research criteria for the diagnosis of Alzheimer's disease: revising the NINCDS-ADRDA criteria. Lancet Neuro/ 6, 734-746, doi:10.1016/s1474-4422(07)70178-3 (2007).

9. Folstein, M. F., Folstein, S. E. \& McHugh, P. R. "Mini-mental state". A practical method for grading the cognitive state of patients for the clinician. $J$ Psychiatr Res 12, 189-198, doi:10.1016/0022-3956(75)90026-6 (1975).

10. Zalewska, A., Szarmach, I., Zendzian-Piotrowska, M. \& Maciejczyk, M. The Effect of N-Acetylcysteine on Respiratory Enzymes, ADP/ATP Ratio, Glutathione Metabolism, and Nitrosative Stress in the Salivary Gland Mitochondria of Insulin Resistant Rats. Nutrients 12, 458, doi:10.3390/nu12020458 (2020).

11. Navazesh, M., Christensen, C. \& Brightman, V. Clinical criteria for the diagnosis of salivary gland hypofunction. J Dent Res 71, 1363-1369, doi: 10.1177/00220345920710070301 (1992).

12. Zalewska, A. et al. Rheumatoid arthritis patients with xerostomia have reduced production of key salivary constituents. Oral Surg Oral Med Oral Pathol 115, 483-490, doi: 10.1016/j.0000.2012.10.013 (2013).

13. Misra, H. P. \& Fridovich, I. The role of superoxide anion in the autoxidation of epinephrine and a simple assay for superoxide dismutase. $J$ Biol Chem 247, 3170-3175 (1972).

14. Aebi, H. Catalase in vitro. Methods in Enzymol 105, 121-126, doi: 10.1016/s0076-6879(84)05016-3 (1984).

15. Mansson- Rahemtulla, B., Baldone, D. C., Pruitt, K. M. \& Rahemtulla, F. Specific assays for peroxidases in human saliva. Arch Oral Bio/31, 661-668, doi: 10.1016/0003-9969(86)90095-6 (1986).

16. Griffith, O. W. Determination of glutathione and glutathione disulfide using glutathione reductase and 2-vinylpyridine. Anal Biochem 106, 207-212, doi: 10.1016/0003-2697(80)90139-6 (1980).

17. Erel, O. A novel automated direct measurement method for total antioxidant capacity using a new generation, more stable ABTS radical cation. Clin Biochem 37, 227-285, doi: 10.1016/j.clinbiochem.2003.11.015 (2004).

18. Erel, O. A new automated colorimetric method for measuring total oxidant status. Clin Biochem 38, 1103-1111, doi: 10.1016/j.clinbiochem.2005.08.008 (2005).

19. Kołodziej, U. et al. Chronic high-protein diet induces oxidative stress and alters the salivary gland function in rats. Arch Oral Bio/ 84, 6-12, doi:https://doi.org/10.1016/j.archoralbio.2017.09.006 (2017).

20. Kalousová, M., Skrha, J. \& Zima, T. Advanced glycation end-products and advanced oxidation protein products in patients with diabetes mellitus. Physiological research 51, 597-604, (2002).

21. Buege, J. A. \& Aust, S. D. Microsomal lipid peroxidation. Methods Enzymo/ 52, 302-310, doi: 10.1016/s0076-6879(78)52032-6 (1978).

22. Grisham, M. B., Johnson, G. G. \& Lancaster, J. R. Quantitation of nitrate and nitrite in extracellular fluids. Methods Enzymo/ 268, 237-246, doi: 10.1016/s0076-6879(96)68026-4 (1996).

23. Choromańska, B. et al. Bariatric Surgery Normalizes Protein Glycoxidation and Nitrosative Stress in Morbidly Obese Patients. Antioxidants (Basel, Switzerland) 9, 1087, doi:10.3390/antiox9111087 (2020). 
24. Beckman, J. S. et al. Kinetics of superoxide dismutase- and iron-catalyzed nitration of phenolics by peroxynitrite. Arch Biochem Biophys 298, 438445, doi: 10.1016/0003-9861(92)90432-v (1992).

25. Zalewska, A. et al. NAC Supplementation of Hyperglycemic Rats Prevents the Development of Insulin Resistance and Improves Antioxidant Status but Only Alleviates General and Salivary Gland Oxidative Stress. Oxid Med Cell Longev 2020, 8831855, doi:10.1155/2020/8831855 (2020).

26. Pawlukianiec, C. et al. A New Insight into Meloxicam: Assessment of Antioxidant and Anti-Glycating Activity in In Vitro Studies. Pharmaceuticals (Basel) 13, 204, doi:10.3390/ph13090240 (2020).

27. Ship, J. A., DeCarli, C., Friedland, R. P. \& Baum, B. J. Diminished submandibular salivary flow in dementia of the Alzheimer type. J Geronto/ 45, M6166, doi:10.1093/geronj/45.2.m61 (1990).

28. Proctor, G. B. \& Carpenter, G. H. Regulation of salivary gland function by autonomic nerves. Auton Neurosci 133, 3-18, doi:10.1016/j.autneu.2006.10.006 (2007).

29. Jann, M. W. Rivastigmine, a new-generation cholinesterase inhibitor for the treatment of Alzheimer's disease. Pharmacotherapy 20, 1-12, doi:10.1592/phco.20.1.1.34664 (2000).

30. Sayer, R., Law, E., Connelly, P. J. \& Breen, K. C. Association of a salivary acetylcholinesterase with Alzheimer's disease and response to cholinesterase inhibitors. Clin Biochem 37, 98-104, doi:10.1016/j.clinbiochem.2003.10.007 (2004).

31. Thanan, R. et al. Oxidative stress and its significant roles in neurodegenerative diseases and cancer. Int J Mol Sci 16, 193-217, doi:10.3390/ijms16010193 (2014).

32. Zieniewska, I., Maciejczyk, M. \& Zalewska, A. The Effect of Selected Dental Materials Used in Conservative Dentistry, Endodontics, Surgery, and Orthodontics as Well as during the Periodontal Treatment on the Redox Balance in the Oral Cavity. Int J Mol Sci 21, 9684, doi:10.3390/ijms21249684 (2020).

33. Maciejczyk, M. et al. Antioxidant Defense, Oxidative Modification, and Salivary Gland Function in an Early Phase of Cerulein Pancreatitis. Oxid Med Cell Longev 2019, 8403578, doi:10.1155/2019/8403578 (2019).

34. Hipkiss, A. R. Accumulation of altered proteins and ageing: causes and effects. Exp Gerontol 41, 464-473, doi:10.1016/j.exger.2006.03.004 (2006).

35. Reznick, A. Z., Klein, Y., Eisfrich, J. P., Cross, E. C. \& Nagler, R. M. Inhibition of oral peroxidase activity by cigarette smoke: in vivo and in vitro studies. Free Rad Biol Med 34, 377-384, doi: 10.1016/s0891-5849(02)01297-2 (2003).

36. Zalewska, A. et al. Antioxidant profile of salivary glands in high fat diet- induced insulin resistance rats. Oral diseases 20, 560-566, doi: 10.1111/odi.12173 (2014).

37. Atwood, C. S. et al. Characterization of copper interactions with alzheimer amyloid beta peptides: identification of an attomolar-affinity copper binding site on amyloid beta1-42. J Neurochem 75, 1219-1233, doi:10.1046/j.1471-4159.2000.0751219.x (2000).

38. Schaar, C. E. et al. Mitochondrial and cytoplasmic ROS have opposing effects on lifespan. PLoS Genet 11, e1004972, doi:10.1371/journal.pgen.1004972 (2015).

39. Hopkins, R. Z. \& Li, R. Y. Essential of free radical biology and medicine. (Cell Med Press AlMSCl, Inc., 2017).

40. Cioffi, F., Adam, R. H. I. \& Broersen, K. Molecular Mechanisms and Genetics of Oxidative Stress in Alzheimer's Disease. J Alzheimers Dis 72, 9811017, doi:10.3233/jad-190863 (2019).

41. Zhang, Y. J. et al. Peroxynitrite induces Alzheimer-like tau modifications and accumulation in rat brain and its underlying mechanisms. FASEB journal : official publication of the Federation of American Societies for Experimental Biology 20, 1431-1442, doi:10.1096/fj.05-5223com (2006).

42. Di Domenico, F., Barone, E., Perluigi, M. \& Butterfield, D. A. The Triangle of Death in Alzheimer's Disease Brain: The Aberrant Cross-Talk Among Energy Metabolism, Mammalian Target of Rapamycin Signaling, and Protein Homeostasis Revealed by Redox Proteomics. Antioxidants \& redox signaling 26, 364-387, doi:10.1089/ars.2016.6759 (2017).

43. Reinheckel, T. N., B. et al. Occurrence of oxidatively modified proteins: an early event in experimental acute pancreatitis. Free Rad Biol Med 24, 393400, doi: 10.1016/s0891-5849(97)00271-2 (1998).

44. Adamczyk-Sowa, M. et al. Oxidative modifications of blood serum proteins in myasthenia gravis. Journal of neuroimmunology 305, 145-153, doi:10.1016/j.jneuroim.2017.01.019 (2017). 